\title{
Toward a ubiquitous virtual collaboration environment: A fusion of traditional and leading-edge virtualization tools that empower distributed participants to explore, discover and exchange information without traditional boundaries or constraints
}

\author{
Tony Vanchieri *, Luke Sebby and Gary Dooley \\ Virginia Tech Applied Research Corporation, as the Partnership Intermediary for the Air Force Office \\ of Scientific Research, 900 North Glebe Road, Arlington, VA 22203, USA \\ E-mails: \{tony.vanchieri, Luke.Sebby, Gary.Dooley\}@vt-arc.org
}

\begin{abstract}
The Virginia Tech Applied Research Corporation (VT-ARC) - a private, non-profit corporation affiliated with Virginia Tech - was selected by the Air Force Office of Scientific Research (AFOSR) to create a Basic Research, Innovation, and Collaboration Center. The Center will provide the Air Force an innovative and collaborative environment accessible to academia, industry and other government entities. The BRICC collaborative services and environment will assist AFOSR in evolving from "doing things better" to "doing things differently" with respect to Basic Research Initiatives, Multidisciplinary University Research Initiatives, Small Business Technology Transition, workforce development including Science, Technology, Engineering, and Math (STEM) programs, and international collaboration.
\end{abstract}

Keywords: Scientific collaboration, virtual collaboration, basic research

\section{Basic Research, Innovation and Collaboration Center (BRICC)}

The Air Force Office of Scientific Research (AFOSR), located in Arlington, Virginia, continues to expand the horizon of scientific knowledge through its leadership and management of the Air Force's basic research program. As a vital component of the Air Force Research Laboratory (AFRL), AFOSR's mission is to discover, shape, and champion basic science that profoundly impacts the future Air Force. The focus of AFOSR is on research areas that offer significant and comprehensive benefits to our national warfighting and peacekeeping capabilities. These areas are organized and managed in three scientific directorates: Aerospace, Chemical and Material Sciences; Mathematics, Information and Life Sciences; and Physics and Electronics.

\footnotetext{
*Corresponding author. Tel.: +1 5714228503 (cell), +1 5713843808 (office); E-mail: tony.vanchieri@ vt-arc.org.
} 
In its pursuit of revolutionary basic research, AFOSR awards nearly 1,500 grants and contracts to more than 200 academic institutions, 160 commercial firms, and more than 250 intramural programs within the nine other AFRL technology directorates. With its staff of highly trained scientists and engineers, AFOSR manages the Air Force basic research program via three key partnerships including the university connection, small business technology transfer, and Air Force intramural research. A broad and complex agenda, AFOSR requires a modern, state-of-the art innovation and collaboration center to maintain its position as the revolutionary basic science and technology organization of the future.

In December 2012, AFOSR established the requirement for a Basic Research Innovation and Collaboration Center (BRICC) to enhance its mission in innovative research. The BRICC was envisioned to include an innovative and collaborative environment accessible to AFOSR, academia, industry and other government entities in the Washington DC area. AFOSR's goal is to leverage the capabilities of the BRICC to evolve from "doing things better" to "doing things differently" with respect to AFOSR's Basic Research Initiatives (BRI), Multidisciplinary University Research Initiatives (MURI), Small Business Technology Transition, Science, Technology, Engineering, and Math (STEM) programs, and international collaboration. In doing things differently, AFOSR wanted to establish a new, innovative partnership with an unbiased, neutral facilitator, who could act as the Partnership Intermediary on behalf of AFOSR's basic research, innovation, outreach and collaboration goals.

AFOSR selected the Virginia Tech Applied Research Corporation (VT-ARC, http://vt-arc.org) and awarded a Partnership Intermediary Agreement (PIA) under the Department of Defense (DoD) Grant and Agreement Regulations (DoDGARS) Part 32 and Part 34 to establish and facilitate a BRICC. ${ }^{1}$ VT-ARC is a private, non-profit, university affiliated research corporation, with offices in Arlington and Blacksburg, Virginia. VT-ARC, in collaboration with stakeholders, develops innovative solutions to complex national challenges by drawing upon the rich multidisciplinary research community including private and public sector partners.

As the Portal to the Power of Virginia Tech, VT-ARC integrates and unites interdisciplinary capabilities across a spectrum of AFOSR research partners and stakeholders. VT-ARC will be supporting AFOSR along two key focal areas: collaboration services and collaboration environment. The collaboration services represent a unique suite of engaging, interactive workshops and other collaborative-style events, tailored to AFOSR specific needs. The innovation engine and creative catalyst for these activities is the rich discourse resulting from the interdisciplinary perspectives and contributions of participants from government, academia and industry alike. BRICC services aim to support AFOSR in thinking creatively, collaboratively and most importantly, differently as it pursues revolutionary basic research.

Bolstered by the collaborative exchanges among its global network of entrepreneurs, academicians, small business owners, scientists and engineers, BRICC services provide creative and innovative solutions to AFOSR challenges across a spectrum of focal areas as shown in Fig. 1. Among the various BRICC collaboration services are:

- Future Visioneering Series - an ideation event focused on anticipating the next revolution in basic scientific research that will impact the Air Force.

\footnotetext{
${ }^{1}$ Within DoDGARS is a category of agreement, known as a PIA, between DoD and a non-profit organization, for the purposes of facilitating basic research, advanced research, and technology transition/transfer. A PIA is a "Technology Transfer Mechanism" between the government and a nonprofit entity that allows the Partnership Intermediary (VT-ARC) to:

- identify new technologies in the private sector that can be utilized by the government,

- facilitate joint projects between the government and private companies, as well as between agencies and academic institutions, to accelerate delivery of technological capabilities to the nation,

- help existing companies identify government technologies that can be licensed and commercialized.
} 


\section{STEM and Workforce Development}
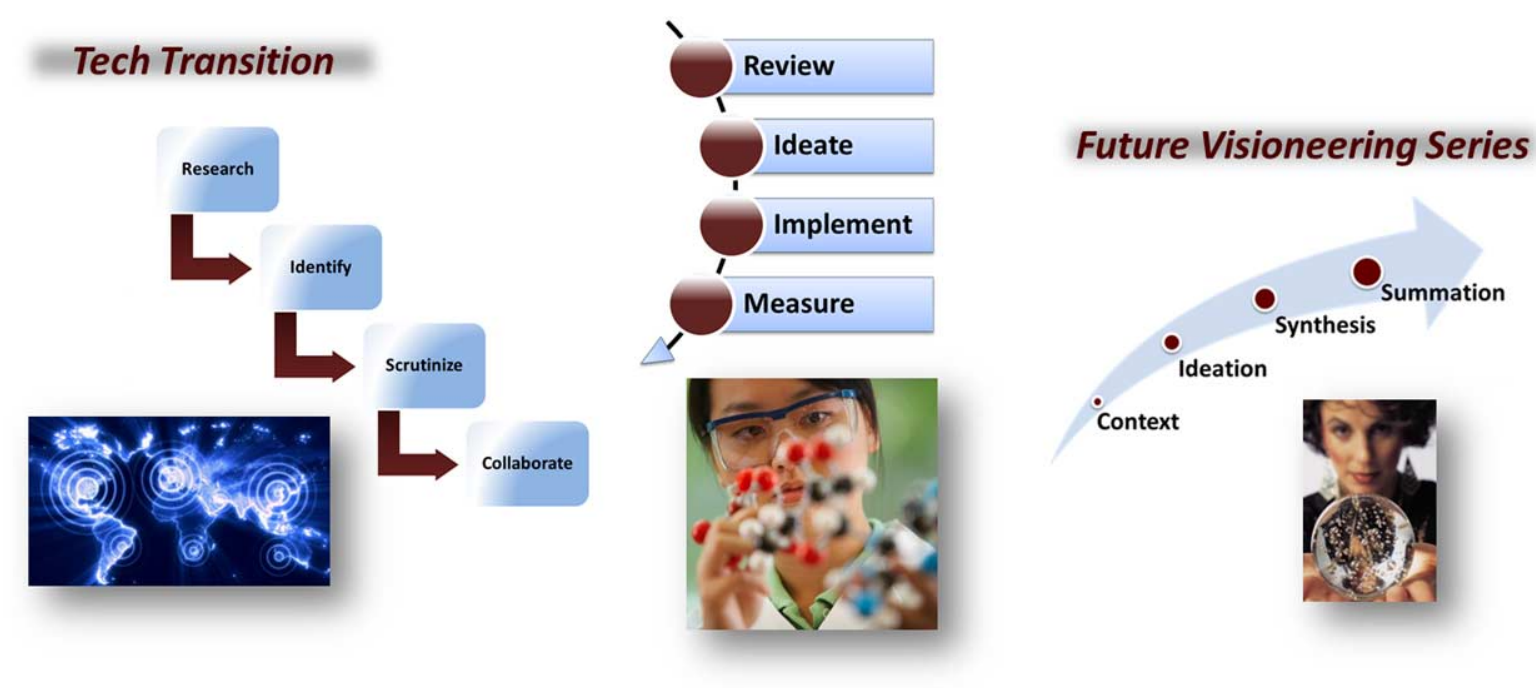

Fig. 1. Innovative facilitated collaborative services. (Colors are visible in the online version of the article; http://dx.doi.org/ 10.3233/ISU-130716.)

- Workforce Development Workshops - collaborative exchanges engaging AFOSR in a multidisciplinary strategic dialogue on STEM and its scientific cadre.

- Small Business Interchanges - interactive tech transition events introducing the AFOSR basic research portfolio to small businesses and entrepreneurs.

The BRICC holds other collaborative events as well including entrepreneurial challenges to engage the small business community and spark technology transfer opportunity. Each of these collaborative events targets a unique section of AFOSR's strategic agenda such as the future allocation of resource for basic scientific research; ensuring a steady stream and cadre of educated and experienced scientists; and the transition and transfer of 6.1 basic scientific research to applied research and commercialization.

Collaborative services are impossible without the rich collaborative ecosystem provided by the BRICC's collaborative environment. To support the collaborative ecosystem VT-ARC simultaneously leverages existing collaborative technologies in both traditional means as well as simultaneously combining those technologies in new ways to facilitate collaboration to all audiences increasing the collaborative capabilities on all levels.

As seen in Fig. 2, the current collaborative technologies used include: ${ }^{2}$

- VTC - Leveraging HD cameras, real time encoding as well as multi-framework web protocol capabilities ensures high quality communication between endpoints anywhere on the globe.

- Web Collaboration Frameworks - By using multiple web collaboration technologies simultaneously VT-ARC is able to ensure all participants are able to contribute on many levels (chat, white boarding, document sharing and editing as well as multilevel presentations).

- VOIP Tele-conferencing - Used as not only a direct communication tool, Voice Over IP can be used to facilitate one off collaboration where direct en masse collaboration is less ideal. Many VOIP

\footnotetext{
${ }^{2}$ Trademarks or images listed herein are representative for illustrative purposes only, and belong to their respective owners.
} 


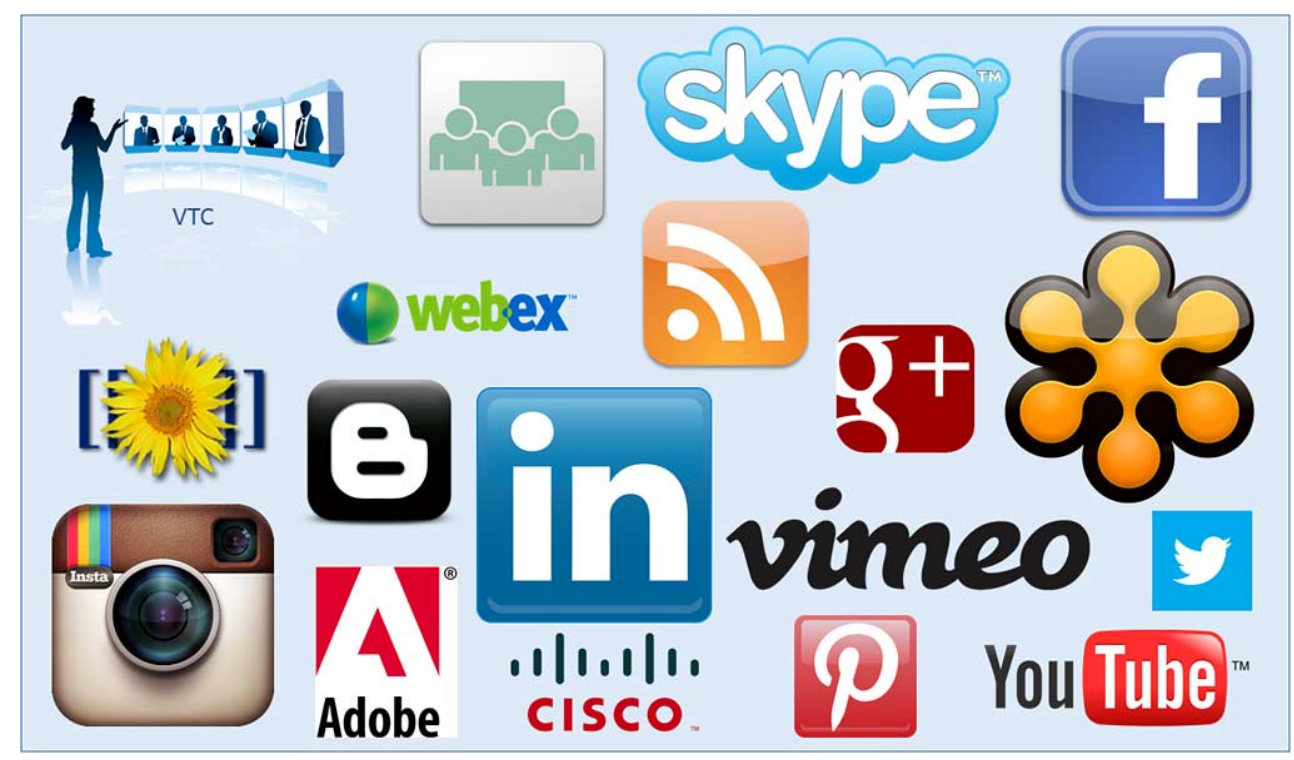

Fig. 2. The BRICC will integrate collaborative tools into a ubiquitous virtual environment. (Colors are visible in the online version of the article; http://dx.doi.org/10.3233/ISU-130716.)

applications offer many of the same functional capabilities as the web collaboration frameworks however usually on a much smaller scale.

- Real time streaming - Where endpoint to endpoint ends, real time streaming collaboration begins. By leveraging not only the one to many capabilities of streaming, other streaming functions increase collaboration trough viewer feedback, question and answer sessions, along with participant polling and end session surveys.

- On-Demand Video - Long after the events have ended multitudes may still contribute to the collaboration by not only consuming the original event but by tagging, commenting and providing feedback to the originators of the content.

- Live Data Feeds - For those unable to attend either the physical event or the virtual session, others may still participate by receiving a live data stream produced during the event through multiple different sources including RSS feeds, real time blogs and live audio.

- Social Networking Integration - Leveraging public social media such as Twitter, Facebook, LinkedIn, Pinterest, Tumblr, Google+ and others, collaboration can be presented to the entire world which in turn creates new collaborators who contribute in new ways yet to be thought of today.

Alone, none of these collaborative technologies pushes the envelope to facilitate greater communication and discovery. However but integrating these technologies and utilizing their individual strength in concert, a truly powerful collaborative platform emerges. This integration is provided through a web framework called the Portal for Research, Technology Exposure and Outreach (PoRTEO), an extensible platform designed to not only integrate the above technologies but also provides mechanisms for reaching out to individual and groups, bringing them together to begin the collaborative process.

Taken together, the Collaboration Services and Collaboration Environment merge to form a ubiquitous virtual collaborative environment (UVCE) that spans organizational boundaries and facilitates collaboration, outreach, research, and experimentation in both the continental United States (CONUS) and outside the continental United States (OCONUS). The UVCE is a fusion of the traditional physical col- 
laboration environment (meeting spaces) and leading-edge virtualization tools that empower distributed participants to explore, discover, and exchange information without traditional boundaries or constraints. UVCE is also a knowledge discovery and management capability, where complex challenges and technologies are deconstructed to their basic elements for increased observability, understanding, utility, and re-use. The UVCE unites both the challenge space and the solution space. It spans key aspects to include: people, strategies, policies, tools, processes, best practices, operations, technology, goals, objectives, constraints, costs and related attributes. The UVCE enhances collaboration and effective technology development by involving others within a global research community through a broad and effective virtual collaboration architecture. Further, the UVCE's knowledge management capability will collect, store, archive, index, and make available to the public, the results of various collaborative activities undertaken by VT-ARC and the BRICC.

Figure 3 depicts the relationship between stakeholders and collaboration capabilities. As the number of stakeholders increases, the collaboration architecture must drive toward an integrated sophisticated suite of collaboration tools, both virtual and physical, to facilitate ubiquitous collaboration. The UVCE will allow AFOSR to more effectively communicate, understand, and interact with industry, academia, and other government agencies in basic research development, both nationally and internationally, to foster and enhance technology transfer opportunities. VT-ARC will leverage sophisticated virtual collaboration tools that enable one-to-many, and many-to-many collaboration, communication, and information exchanges on a global scale. Moreover, VT-ARC will provide for multidisciplinary forums, and will assist AFOSR in creating and maintaining a knowledge transition environment, where Basic Research is guided along a transition path to opportunities for Applied Research and Advanced Technology Development. In addition to technology transition, VT-ARC will foster awareness of and interest in transfer of U.S. Government (USG) science and technology to the private sector for commercialization and economic development. For initial physical collaboration, VT-ARC will provide AFOSR and its partners, a modern, state-of-the-art conference and meeting facility in which to engage in intense collaborative

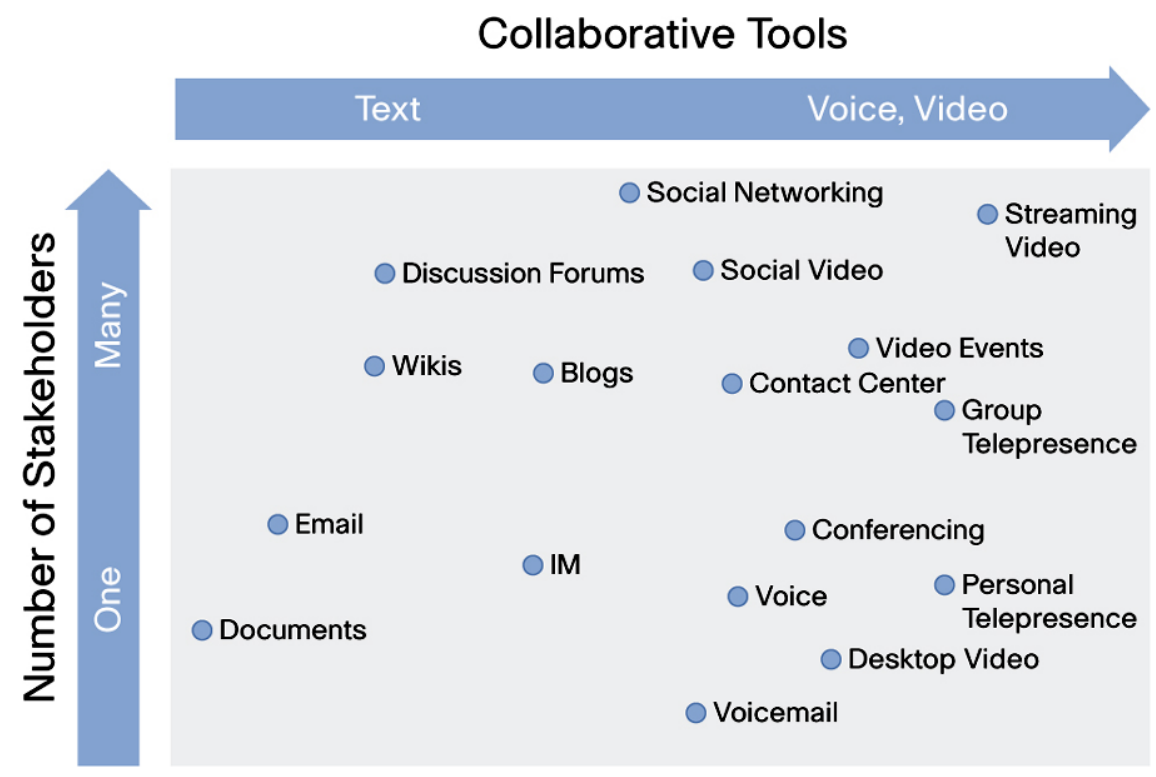

Fig. 3. The evolution of collaboration tools as complexity grows. (Colors are visible in the online version of the article; http://dx.doi.org/10.3233/ISU-130716.) 
sessions. Physical collaborations will be recorded, archived, indexed, and made available to the public, through social media or other collaborative means.

The collaboration ecosystem includes maintaining, refining, and demonstrating the processes, facilities, and partnerships necessary for AFOSR to effectively employ an innovation and collaboration environment that is accessible to AFOSR, academia, industry and other government entities. The collaboration ecosystem will evolve to be a persistent resource for collaboration and innovation that encourages creative exploration, understanding, and exploitation of basic research. Consistent within this scope are the following objectives/goals:

- Facilitating collaboration - develop and operate a collaboration infrastructure that supports planned and $a d-h o c$ information exchanges, either physically or virtually. The collaboration infrastructure will make use of advanced, virtual collaboration, knowledge discovery and knowledge exploitation tools. Collaborations will be available to partners CONUS and OCONUS, and material developed or generated during these collaboration sessions will be indexed, archived, and made available to AFOSR partners.

- Assisting AFOSR with strategic management of its basic research and science portfolio - assist AFOSR in review and development of its basic research portfolio, to include BRIs and MURIs. The review and development includes providing recommendations to AFOSR's basic research portfolio in terms of mix of projects, turnover, and methods to determine efficiencies within the overall portfolio.

- Facilitating partnerships - assist AFOSR in developing robust national and international Science and Technology (S\&T) partnerships with government, academia and industry organizations; as well as partnerships with other State, National or other public institutions to leverage knowledge transition and/or resources that support AFOSR.

- Knowledge transfer/transition - assist AFOSR in creating and maintaining a knowledge transition environment, where Basic Research is shepherded along a transition path to Applied Research and Advanced Technology Development, as appropriate. VT-ARC will ensure AFOSR and its collaboration partners have access to innovation tools, methodologies, and problem analysis approaches to characterize, qualify, and develop metrics to quantify and objectively measure, the success of this knowledge transfer/transition.

- Technology transition - identify, develop, and where possible exploit opportunities to accelerate the transition of technology to select mission applications, operational environments, urgent operational needs, high impact challenge areas, and non-traditional applications (including commercial applications). Technologies will include both materiel and non-materiel components including (but not limited to) tactics, techniques, processes and procedures. Identification and development of transition opportunities may include studies and analyses, test and evaluation activities. Attract technology transition customers from industry to foster accelerated commercialization and value enhancement of government-funded research.

- Technology transfer - identify and develop capabilities and tools to assist in transferring USG technology from the Government/public sector to the private sector for commercialization and economic development.

- "Future Visioneering" - engage AFOSR in "future visioneering" exercises - periodic, deliberate and creative examinations of future trends in S\&T, and how to best position AFOSR to lead the way.

- International collaboration and facilitation - extend the collaboration and innovation support to AFOSR detachments worldwide. 
Through the BRICC, VT-ARC will provide an innovative collaborative environment, integrate emerging collaborative technologies, and provide neutral facilitation to bring together the right mix of academia, industry and government to address challenging Air Force problems. The BRICC will embody the people, services, and activities for government, industry, and academia and other partners, to meet and work in collaborative teams, workshops and forums. This partnership will enhance the technology transition and transfer of Air Force basic Research results and support STEM efforts to develop and attract the next generation of scientists and engineers in critical Air Force research areas.

Mr. Tony Vanchieri will lead VT-ARC's role to enhance the connections between the Air Force, academia, industry and other government agencies to identify and exploit basic research opportunities that will impact the future Air Force.

For further information, please visit:

AFOSR: http://www.wpafb.af.mil/afrl/afosr/,

VT-ARC: http://vt-arc.org/.

VT-ARC's Technology partner in instantiating elements of the collaborative environment is NFF. NFF is an IT Solutions provider, specializing in Data Center Virtualization, Unified Communications, Network Infrastructure, Information Security and Professional Services. NFF's proficiency and experience allows customers to depend on a single source for planning, deployment and long-term support of complex projects. NFF: http://nffinc.com/. 